\title{
Le silence de mort du syndrome de Münchhausen par procuration
}

\author{
Catherine Roudot-Granaux, docteur en psychopathologie clinique, psychologue clinicienne, \\ 10 rue de Nesle, 75006 Paris - catherine.granaux@dartybox.com \\ Mareike Wolf-Fédida, Professeur des Universités et psychanalyste, UFR d'Études \\ psychanalytiques, université Paris Diderot - Paris 7, bâtiment Olympe de Gouges - case \\ courrier 7058, 8 rue Albert Einstein, 75205 Paris Cedex 13 -M14Wolf@aol.com
}

Le terme de syndrome de Münchhausen par procuration désigne un trouble factice ou non au cours duquel un parent, la mère généralement, produit ou simule chez l'enfant des symptômes ou une maladie dans le but de lui faire jouer le rôle du malade, puis le présente aux médecins pour qu'il soit soumis à des traitements et à des examens multiples.

Le DSM-IV ${ }^{1}$ ne considère pas le syndrome de Münchhausen comme une maladie mentale. Il le définit comme une production intentionnelle où le fait de feindre des signes physiques chez une personne dont on prend soin se fait dans le but de lui faire jouer le rôle du malade. Dans ce syndrome, la perception du caractère malfaisant est quasiment toujours présente et apparaît indirectement évidente par le côté prémédité et simulé de ces faits, sinon, les méfaits ne seraient pas perpétrés de manière déguisée et secrète. Cette forme grave de maltraitance est caractérisée par une double distorsion de la triade classique en pédiatrie : parent-enfant-médecin. Dans le syndrome de Münchhausen par procuration, les mères le plus souvent occupent le devant de la scène. Le père n'intervient pas entre la mère et l'enfant. Les auteurs s'accordent pour dire que le père est faible, voire absent.

Parallè lement, la mère accorde peu de valeur à celui-ci, préoccupée par les allégations qui dissimulent une problématique narcissique. En effet, la mère exerce son action sur l'enfant, mais aussi influence et manipule le corps médical. Les médecins prescrivent des examens complémentaires, des bilans invasifs voire des interventions chirurgicales inutiles et même dangereuses pour l'enfant, jouant un rôle important dans la genèse et la durée de ce syndrome, se détournant à leur insu et donc involontairement de leur fonction soignante. C'est donc la conséquence d'un double mécanis me de maltraitance, à savoir l'action conjuguée du parent et de médecins .

Objet fétiche de la mère, l'enfant déshumanisé ne peut élaborer correctement son identité puisqu'il donne corps au désir de sa mère et n'a pas d'existence propre. Aussi pourrait-on voir dans la dépression maternelle, une indistinction Moi-objet. La notion de personne même disparaît ${ }^{2}$. Ces mères ne s'aperçoivent pas qu'elles ont un petit effectivement vivant. L'enfant est utile dans une fonction qui peut conduire jusqu'au sacrifice. On peut imaginer que ces mères, enfants déjà sacrifiés, attaquent ainsi une partie de leur propre corps.

\section{MÉTHODOLOGIE}

Exerçant en libéral en tant que psychologue clinicienne, nous avons reçu en thérapie une patiente victime du syndrome de Münchhausen par procuration, ayant vécu toute son enfance de multiples interventions chirurgicales et investigations médicales sous contrainte maternelle. L'histoire de Sylvie nous a permis d'analyser les interactions familiales et du fait qu'elle-même a entraîné sa fille dans une spirale médicale, ses propres réactions face aux médecins.

Nous allons donc tenter de mettre à jour dans ce travail les tenants de ces rencontres ainsi que les hypothèses concernant les éléments d'une structure psychique pathologique en exposant la manière dont elle se déploie, car le nœud de la problématique du patient émerge dans l'observation d'un comportement familial. Ce cycle comportemental donne ainsi les éléments d'une réflexion sur le problème de la filiation et de ce que la patiente répète, au-delà de son comportement relationnel, d'une

\footnotetext{
1 DSM-IV-TR, Manuel diagnostique et statistique des troubles mentaux, Issy-les-Moulineaux, ElsevierMasson, 2002.
}

2 A. Le Nestour, A.M. Raphaël, S. Raphäl, G. Golberine, « Infanticides sous surveillance médicale 
position familiale.

Rescapée, c'est ainsi que se qualifie cette femme réservée d'une quarantaine d'années. Elle pense que si les médecins avaient été au courant de son calvaire, tout cela n'aurait pas duré aussi longtemps. Elle s'est mariée à 31 ans, avec son mari, psychiatre, son aîné de quinze ans, pour avoir l'impression de se protéger de la folie et aussi parce qu'elle se sent attirée, elle aussi, par les médecins. Ils ont deux filles. Depuis son mariage, elle a pardonné beaucoup de choses, et ressent de la pitié pour sa mère. Elle a été déprimée jusqu'à la fin de la première psychothérapie, qui s'est terminée en 2002, puis a rechuté. Elle se présente donc pour une dépression et un sentiment d'insécurité.

Elle a trois frères, dont deux sont morts, adultes, suite à un cancer, faute de consultations médicales. C'est la benjamine d'une famille aisée. La mère, que nous appellerons $\mathrm{Mme}_{\mathrm{me}} \mathrm{M}$., est une belle femme, qui ne travaille pas et appartient à la classe moyenne. Les relations sociales de $\mathrm{Mme} \mathbf{M}$. sont pauvres, le désœuvrement est marquant. Mme M. a souffert pendant la guerre. Elle a été battue par sa mère et ses deux frères, très durs avec elle, qui la martyrisaient verbalement et physiquement mais, n'étant jamais malade, elle n'aurait donc pas connu d'acharnement médical maternel. Le père était autoritaire, la mère était plus redoutable encore ; «mon père tre mblait devant elle », dira Sylvie.

La mère de Sylvie est décrite comme intrusive et exclusive, entourée de médicaments, de tensiomètres et de thermo mètres. Elle a tendance à boire et à s'automédiquer. Elle a fait venir durant l'enfance et l'adolescence de Sylvie des médicaments de pays étrangers, par relation. Ses connaissances médicales sont bonnes et le «Vidal» est un de ses livres préférés. Actuellement, Sylvie voit sa mère une fois par semaine et téléphone tous les jours, sous peine de chantage : sa mère menace de se suicider. L'emprise est donc toujours effective.

Les souvenirs de Sylvie commencent à 8 ans, âge où elle se rappelle des premiers examens médicaux, qui perdureront jusqu'à l'adolescence. Tout a commencé avec l'appendicite. Ce sera le point de départ d'une longue série d'examens en tous genres, car un excès d'acide urique a provoqué des crises de goutte. Sylvie est aussi atteinte à 10 ans d'obésité due à la cortisone admin istrée à long terme par sa mère à l'insu des médecins. Déscolarisée, son éducation est assurée par un précepteur qui venait à la maison. La mère de Sylvie a épousé M. M. sans amour. Les revendications vis-à-vis de son mari ont été fréquentes, elle tenait aussi un discours moralisateur et exerçait une emprise violente ou séductrice. Pour elle, les hommes étaient tous obsédés sexuellement. La mère a traumatisé sa fille avec la religion catholique et des images «s sanguinolentes » de crucifixion.

Le profil de $\mathrm{M}_{\mathrm{me}} \mathrm{M}$. correspond bien à celui décrit dans la littérature : des connaissances médicales et une fascination pour la médecine. Elle présente une passion morbide pour tout ce qui a trait à la médecine et survalorise la profession médicale, à laquelle pourtant elle se mesure et se confronte toujours dans une attitude passive-agressive. Durant de longues années, Sylvie a subi de multip les examens méd icaux, une dizaine d'interventions chirurgicales : a mygdalecto mie, appendicite, deux opérations avec anesthésie générale pour des verrues, une intervention chirurgicale pour une aiguille enfoncée dans le pied, kyste à l'aine, kyste à l'ovaire, deux extractions de dents de sagesse, deux interventions gynécologiques, coloscopie, toutes sous anesthésie générale et pour la plupart inutiles, conjuguées à l'administration massive de médicaments, moyennant quoi elle était dédommagée en cadeaux. Mme M. a pu opérer en toute quiétude car le silence de sa fille l'a protégée et Sylvie s'est soumise totalement à elle.

Le père de S., M.M., était fils unique. Il a également souffert de l'emprise de sa mère, qui était très dure avec lui. Adolescent, il fuguait pour échapper à l'autorité parentale. Les parents de M. M. semblaient accaparants au point qu'une fois marié, il accompagnait son père au café et délaissait sa femme. Ses parents se sont sacrifiés pour lui : « des gens méchants », disait la mère de Sylv ie qui se préoccupait essentiellement de faire le vide autour d'elles. La grand-mère paternelle est morte à 78 ans d'un cancer. Jeune femme, elle a eu un accouchement très difficile par le siège avec hémorrag ie, ce qui a provoqué une haine des médecins. C'est aussi pour cette raison qu'elle n'a plus voulu d'autres enfants. Sylvie décrit son père curieusement comme le plus charmant du monde puis peu après comme rig ide et très strict. M. M. parlait peu et jamais de ses parents. Sylvie ne ressentait pas l'amour de sa mère pour ce mari démissionnaire.

D'après ce témoignage, le père était ligoté dans une relation très étroite et intense avec ses parents et apparemment, cherchait peu à intervenir dans la dyade mère-enfant. Sylvie fut alors écrasée par la toute-puissance maternelle. M. M. a fait deux infarctus, puis, hospitalisé de nouveau pour un problème cardio-vasculaire, est retourné à son domicile à la demande expresse de sa femme, qui ne supportait pas l'idée qu'il ne puisse «plus marcher », à entendre au sens propre (comme au sens figuré ?). Sylvie est persuadée que sa mère « s'est occupée de lui » et qu'il en est mort alors qu'elle n'avait que 11 ans. Sylvie souffre d'une dépression importante.

La dépression est phénoménologiquement un retour dans l'inanimé, une rupture du temps comme coupure, un éternel retour. Ce système de défense renferme des idées de mouvement, de 
continuité et d'autofécondation. Le cercle infini des renaissances et la perpétuelle répétition trahissent la prédominance de la fondamentale pulsion de mort.

« Ma mère m'aimait trop, dira-t-elle lors d'une séance, et dans la famille tout le monde marchait au chantage. » C'est ainsi qu'elle perçoit le comportement de maltraitance maternelle. Elle a vu sa mère folle de rage jeter des meubles sur les murs lorsque, une fois devenue adolescente, elle cherchait à s'individualiser. L'emp rise est telle qu'elle ne peut concevoir de se séparer psychiquement de sa mère au risque de la faire mourir.

On assiste donc à l'impossible séparation d'avec un objet narcissique, et non à un véritable travail de deuil, même pathologique. Le deuil de l'objet primaire ne peut se faire afin de préserver l'omnipotence qui est cultivée avec la mère et de parer au $\mathrm{x}$ angoisses de séparation. L'o mniprésence maternelle se soutient au prix de la subjectivité et la place dans une position de suppléant symbolique et incarne un idéal absolu. Mais enfant, elle a été dépossédée de ses pensées, et du plaisir de les investir. On lui a nié tout droit à prétendre vrais les sentiments éprouvés et ses ressentis. Mme M. l'a giflée à plusieurs reprises après avoir fouillé sa chambre de fond en comble et lu la souffrance exprimée dans son journal intime, pourtant dissimulé par prudence sous le linoléum de sa chambre. Le silence a donc été le seul moyen de ne pas assombrir davantage sa vie de recluse. Sylvie voit sa mère une fois parsemaine, c'est un devoir. «Je ne peux pas laisser tomber ma mère, je ne voudrais pas lui faire supporter mon absence. »

\section{PASSAGE À L'ACTE}

Le passage à l'acte maternel pose le problème des rapports entre la pensée et l'action. Le basculement entre l'acte en fantasme et l'acte réel se trouve interrogé, lorsque celui-ci devrait moralement être inhibé par des mécanismes de défense. Il est l'effet d'un mode préoedipien du fonctionnement psychique où prédominent les processus primaires, incapables de tolérer la frustration, de reconnaître l'épreuve de réalité et de freiner la tendance à l'impulsivité. L'acte évacue toute mentalisation et prouve une carence d'élaboration psychique. En ce qui concerne les actes les plus violents, on peut parler d'effondrement narcissique. L'acte représente alors une preuve d'existence et n'est pas le résultat d'une construction psychique. D'où son aspect pulsionnel brutal. La parole porte elle-même les stigmates du trauma ce dont témoignent les effets d'effacement de l'altérité et des dégâts produits par la transmission de traces muettes aux générations suivantes.

Ces fragments psychiques non représentables viennent coloniser la langue jusqu'à la cadavériser en se logeant directement dans le Réel du corps mais hors possibilité de s'inscrire dans la signifiance du langage. La chaîne signifiante est trouée par des bribes de sons et de présence informe qui trament le discours en cherchant à se faire reconnaître. Cependant, ces cris ne font pas appel, ils sont fixés dans une errance qui traduit l'immobilis me psychique. Nous considérons que ces traces ne peuvent passer du Réel du corps au corps de la langue. L'usage d'une langue cadavérisée, c'est-à-dire non habitée par la part vivante de l'humain, non psychisée, est vidée de tout affect et par là même sans destinataire. C'est pourquoi les passages à l'acte de $\mathrm{Mme} M$. s'expriment par un agir au long cours et non par un passage à l'acte lié au débordement des défenses.

Selon M. Wolf-Fédida: «Dans la pathologie de l'agir, toute symbolisation est rejetée par l'action, toute tension mal supportée est aussitôt évacuée : la mise en question narcissique, le conflit identitaire, l'opposition à l'autorité et aux normes, tout en essayant de se conformer à une certaine image de soi, produisent une certaine violence intérieure qui explique la véhémence des irruptions affectives. $»^{3} \mathrm{Ce}$ qui se résume par faire ce qui est interdit.

L'agir, à la fois processus d'autoengendrement et lutte contre l'effondrement dépressif, demeure le signe d'une souffrance psychique majeure qui cherche à s'élaborer, sans y parvenir, dans une mise en acte violente. Par le passage à l'acte, il s'agit en somme de se précipiter dans le " Réel» par le biais du passage à l'acte, pour ne pas avoir à en subir les effets. Cette instabilité motrice devient une façon d'attaquer les systèmes de symbolisation et la traduction des éprouvés en représentations de mots. Les « reliques » qu'elle porte continuellement avec elle participent aussi de cette tentative de faire figurer le vide représentationnel et l'interdit de penser. Ainsi, en s'identifiant de façon massive à l'objet de la perte, la mère de Sylvie choisit d'incarner le deuil dans sa chair et dans son esprit.

Captif de cette dynamique mutilante, l'agresseur se trouve dès lors incapable de lier ses effractions pulsionnelles à travers un contenant représentationnel, ce qui le contraint à les décharger en permanence dans un présent sans profondeur ni durée, par le biais d'un agir opératoire et dévitalisé. L'agir est pour la mère un moyen de renversement de ce qu'elle craint de subir et de reprendre une

3 M. Wolf-Fédida, Amour, identité et changement, Paris, MJw Fédition, 2005, p. 152. 
maîtrise qu'elle est en train de perdre. L'acte est alors le moyen de figurer sur la scène externe, celle de l'espace environnant, et par là de contrôler ce qu'elle ne peut représenter au niveau d'un Moi sidéré par la massivité des émotions et d'un espace psychique effacé où le jeu subtil de la nomination des émotions et des compromis est impossible. La métaphore du vampire apparaît incarner au mieux le cœur de l'emprise et le mythe de l'irreprésentable. Le crime psychique de l'autre aide les agresseurs à exister psychiquement tandis que les victimes se mutilent psychiquement pour exister, ayant recours au masochisme primaire utilisé comme protection vitale contre l'envahis sement psychique.

Comme nous l'avons vu, le père par sa passivité est caractéristique du couple morbide. Dans la majorité des cas, l'enfant reste exposé aux agirs maternels. "Pour les équipes médicales, le plus frappant est le contraste entre un diagnostic de maltraitance et une attitude maternelle aimante et dévouée envers l'enfant.» ${ }^{4}$

Dans ce syndrome, Sylvie reste liée narcissiquement à la mère, comme objet appartenant à son corps propre. L'ambivalence maintenue dans une relation fusionnelle apparaît au premier $\mathrm{p}$ lan. La duplicité maternelle, fruit du clivage, la conduit à attaquer le mauvais objet que l'enfant vient représenter tout en prétendant agir au nom de son bien. La perte des limites tant psychique que corporelle est réelle et l'adhésivité est déterminante avec la mère, murées toutes les deux dans un secret, dans la collusion du déni, dans la répression de son expression et dans leurs capacités propres de représentation. L'édification d'une crypte se produit quand un secret inavouable et entaché de honte lie le sujet à l'objet ${ }^{5}$. La thématique du secret, si présente chez elles, autorise à penser qu'il y a sans doute en effet dans sa famille un impensé qui se transmet de génération en génération à la manière d'un irreprésentable qui hante ceux qui ne peuvent y donner du sens.

\section{IDENTIFICATION AU MORT}

Non seulement la mère vampirique pose un interdit d'autonomie (dépendance toujours signifiée par les symptômes et les soins) mais elle pervertit le désir d'autonomie par la confusion qu'elle induit. La relation au médecin est faussée d'emblée puisque la mère maîtrise généralement les symptômes. L'absence de sollicitude à l'égard d'un objet total qui serait à la fois bon et mauvais, la dépression de ces sujets ressemblent à une rage impuissante. La mère semble accompagnée d'un objet ancestral dont la présence est d'autant plus envahissante qu'il est hors d'atteinte. L'o mbre de l'ancêtre, le père mort, plane de génération en génération. La dépression est une sorte d'identification à un mort. L'état déprimé se caractérise par des affects archaïques dont le vécu corporel est primordial. « La dépression est un affect dont la caractéristique serait l'altération du temps, la perte de la communication intersubjective et, corrélativement, un extraordinaire appauvrissement de la subjectivité.» $^{6}$

L'identification primitive au mort est telle que la vie psychique devient le tombeau immobile du mort généralement non reconnu et incorporé dans une crypte ${ }^{7}$. La crypte est une sorte d'inconscient artificiel logé au sein du Moi, qui résulte de la perte d'un objet narcissiquement indispensable, et cette perte ne peut pas s'avouer en tant que telle, à cause du secret détenu par le sujet cryptophore. L'hypothèse d'un refoulement conservateur et d'une chaîne se transmettant d'une génération à l'autre, d'un non-dit qui devient pour les enfants porteurs d'un secret encrypté une souffrance irreprésentable. M. Torok $^{8}$ souligne que le trauma de la perte objectale induit [chez le sujet] une réponse : « [...] l'incorporation dans le Moi », elle ajoute néanmoins que « l'objet incorporé, auquel le Moi s'identifie partiellement, rend possible une certaine temporisation en attendant de rééquilibrer l'économie et de redistribuer les investissements ». Ce mode de survie témoigne d'une violence psychique indéniable et le gel du vivant pourrait assurer une sauvegarde contre la psychose. Dans ce sens, on peut donc parler d'homéostasie face aux angoisses d'anéantissement qui submergent Sylvie. La mise à mort dépressive du Moi s'ensuit à la place du matricide ${ }^{9}$. De fait, Sylvie a renoncé à se défendre et s'est retranchée

\footnotetext{
${ }^{4}$ R. Lyons, N. Masters, J. Wu, « Issues in the identification and management of Münchhausen syndrome by proxy », Infant Mental Health Joumal, 12, 4. Michigan, Michigan Association for Infant Mental Health, 1991, 309-320.

5 N. Abraham, M. Torok, L'écorce et le noyau, Paris, Flammarion, 1987, p. 234.

6 P. Fédida, Des bienfaits de la dépression : éloge de la psychothérapie, Paris, Odile Jacob, 2001, p. 10.

7 Ibid., p. 90.

8 Ibid., p. 234.

9 J. Kristeva, Soleil noir : dépression etmélancolie, Paris, Gallimard, 1987 ; coll. « Folio essais », 1989, p. 38-39.
} 
dans un état mort-vivant. La dépression sollicite la création et le génie. Terrible lucidité, elle dévoile qu'en la fin est le commencement et nourrit l'idée de ramener l'orig ine au terme, d'abolir le temps. Aussi cette réflexion nous autorise à considérer qu'il vaut mieux s'immobiliser et s'identifier au père mort plutôt que de survivre dans l'errance d'un monde où le père est forclos. Loin d'être passive, la dépression dispose d'une forme d'activité transformative qui est à penser comme les deux versants d'un mouvement de la pensée, de la vie psychique et de sa mémoire. La matière première du psychisme, mixte de sensorialité et d'éléments phylogénétiques, serait également produite par rapport à la mort et aux morts. Le vivant oscille entre le vivant et de longs moments de stase psychique. Le dépressif est celui qui s'est identifié à l'objet de la perte, celui qui vit dans l'attente éternellement déçue de retrouvailles épiphaniques avec cet Autre, qu'aucun objet ne saurait jamais remplacer, évanoui, entraînant chez le sujet un remords éternel.

Le père mort instaure la mémoire et protège la vie à la condition que le vivant lui reconnaisse une sépulture. Dans la conception de la pulsion d'emprise, ce qui est visé dans la relation, c'est le sujet désirant. Il y a une volonté de neutralisation de l'autre, de réduction de toute altérité. C'est bien ce qui se passe dans le cas de Sylvie avec le recours aux passages à l'acte, masquant la dépression maternelle toujours sous-jacente, qui ne peut jamais combler le sentiment de vide à l'état permanent éprouvé par la mère, et ses passages à l'acte compulsifs. L'interaction sadomasochiste entre la mère et sa fille est déshumanisée : l'enfant est utilisé comme objet fétichiste pour contrôler les relations extérieures. Le tableau vient bien révéler des formes extrêmes de servitude volontaire de tout sujet à l'égard de l'Autre. Cette clinique souligne en effet combien le voeu le plus intime pour Sylvie, attentive à la souffrance maternelle, peut être non pas de se libérer de ses jougs, de se connaître, ou de se réaliser, mais constitue bien plutôt un voeu de servitude vis-à-vis d'elle. Ce souhait, dans des conditions spécifiques, peut se manifester comme demande d'être soulagée de sa propre division, c'est-à-dire de s'abolir comme tel. Selon Maurice Berger : «L'objet fait le mort. » ${ }^{10}$ Rejoindre sa mère serait accepter sa propre disparition au point de laisser l'autre fondre en soi et s'identifier à cet objet perdu.

\section{LES MÉDECINS, TIERS SYMBOLIQUES}

Les médecins ont tendance à croire les allégations des parents, ce qui paraît inévitable en pédiatrie dans la mesure où tous les enfants ne sont pas toujours en âge de s'exprimer ou se taisent sous contrainte. Ils témoignent donc d'une forte résistance à imaginer que les parents puissent chercher délibérément à les tromper. Le médecin représente la loi et un partenaire involontaire parfois dans un jeu avec la mort.

Mme M. cherche à attirer l'attention de l'équipe médicale par sa sollicitude vis-à-vis de l'enfant et à forcer l'admiration de son entourage par son abnégation. Incapable de tisser des liens par ailleurs, la pseudo-maladie infantile lui permet de développer une sociabilité qui lui fait défaut. Cette position est valorisante à plus d'un titre, puisque la mère se sent omnipotente, non seulement en maîtrisant la pathologie et le pronostic de l'affection, mais en plus en ayant le pouvoir de guérir l'enfant du jour au lendemain.

Nous faisons l'hypothèse qu'agissant ainsi, Mme M. essaie de maintenir une relation intense, bien que distante, perverse et ambivalente avec un représentant paternel, avec parfois comme issue désastreuse l'infirmité ou la mort d'un ou de plusieurs enfants. Toute la vie de Mme M. tourne autour de la médecine et elle instaurait un rituel érotique quand le médecin de famille, le seul toléré régulièrement, venait à la maison : bougies, draps en satin étaient les indispensables moyens de séduction de la mère, qui suggérait à sa fille qu'elle avait beaucoup de chance. Les sentiments ambivalents pour le père sont réorientés vers un docteur puis sant et intelligent qui agit sans le savoir en tant que parent symbolique et qui se retrouve mis au défi. L'enfant est alors l'objet d'un soin jaloux et exclusif et tout à la fo is l'objet d'une indifférence surprenante.

La mise en échec du savoir médical, l'image de la mère modèle sont autant de profits psychologiques qui concourent à combler la faille narcissique. Au travers de l'enfant, elles obtiennent enfin la reconnaissance qu'elles n'ont jamais eue, et pourrait-on dire la reconnaissance paternelle qu'elles n'ont jamais connue. Mais ce triomphe permettrait aussi parfois à travers lui de restaurer des relations familiales et de réparer artificiellement l'échec conjugal en introduisant un tiers. Ces mères semblent trouver dans la consultation et plus encore à l'hôpital un lieu d'épanouissement, de «pareangoisse » et de réassurance narcissique. Il s'agit dans la thérapie de trouver la possibilité d'enterrer un père déjà mort par son défaut symbolique, qu'il soit délégué à l'image du tyran despotique, inhumain,

10 M. Berger, «Pathologie du lien primaire et séduction narcissique », Groupal 3, «L'incestuel», Paris, Les Éditions du collège de psychanalyse groupale et familiale, 1997, p. 114-115. 
ou relégué dans l'insouciance d'un père démissionnaire, car les deux ne sont ni identifiables ni attaquables. Le fantasme, présent chez elle, qu'un vivant peut remplacer un mort, fantasme mélancolique s'il en est, qui signifie également qu'elle se sent redevable d'un mort qui ne peut trouver de sépulture et erre dans sa psyché.

L'effacement total des traces du meurtre serait un crime parfait qui remet en scène un père tout-puissant, père de la horde omnipotent, qui n'autorise le lien que par l'entremise de figures despotiques et tyranniques, la mère, dans le cas présent. Ce père induit une résurgence de la jouiss ance sous la forme de meurtres et d'exactions réduits auparavant par la loi primordiale. On peut écrire cette opération à l'inverse de la précédente comme un retour de la jouissance au regard d'une loi bafouée. À 20 ans, ses frères ont bien compris le risque qu'elle encourait et l'ont tirée de force de la maison. Sylvie a fait son sac pendant des semaines en cachette, et ses frères, déjà partis de la maison, lui ont donné rendez-vous en bas dans la rue. Le plus étrange, c'est qu'aucun de ses frères n'a jamais parlé du climat qui y régnait, mais ils se sont tout de même concertés pour la sortir de l'emp rise maternelle :

«C'était un sujet tabou.»

Cette configuration familiale installait des rapports langagiers dominés par le trauma et un silence de mort sur la maltraitance. Sylvie m'explique que peu après, elle a commencé à boire et s'est droguée deux ou trois fois mais que, par peur d'être malade elle s'est arrêtée. Elle a fait plusieurs tentatives de suicide.

\section{TRANSMISSION TRANSGÉNÉRATIONNELLE}

La transmission intergénérationnelle est chose courante dans le syndrome de Münchhausen par procuration. Par rapport à ce qui a été dit précédemment, elle semble même logique, puisque l'enfant n'a pu lui-même acquérir un Moi suffisant pour s'individualiser, ni avoir accès à la symbolisation. Sylvie réalise qu'elle a reproduit le comportement maternel en amenant trop fréquemment sa fille aînée chez le pédiatre. Elle en veut au pédiatre d'avoir eu une attitude qui lui rappelle sa souffrance mais ne se sent pas vraiment coupable. C'est son mari qui a mis fin à ses agissements, conscient de l'engrenage dans lequel elle s'engouffrait. Le mot « mort », n'a pas perdu l'éclat aveuglant et insoutenable du réel non symbolisé, non représentable. Or il semble qu'un tel défaut de symbolisation se soit, dans le cas de Sylvie, transmis de génération en génération, à la man ière d'un impensé qui hante ceux qui l'abritent. Ainsi, si elle ne peut symboliser la mort, et donc s'en séparer, c'est que le deuil éternel dont elle est porteuse est le sien, mais appartient aussi à ses ascendants.

Sylvie et sa mère ont vécu en autarcie, pendant des années dans une mise à l'écart du monde, loin des excitations du monde extérieur et du monde pulsionnel, avec une mise hors-circuit de la rivalité oedipienne, pelotonnées, "comme si c'était un fétiche, sur le secret d'un deuil non fait ». ${ }^{11}$

Une lignée traumatique de narcissisme de mort, dans ce système de filiation narcissique ${ }^{12}$, prédomine. Ce système de filiation narcis sique occasionne chezl'individu qui y adhère de la confusion dans le temps et une perméabilité intergénérationnelle. Il existe une sorte de transmission, en direct, qui n'est plus modélisée par le symbolique. Des choses qui ont eu lieu dans les générations antérieures, notamment la génération précédente, traversent le psychisme du sujet, sans être, en quelque sorte, assimilées. Les souffrances trans générationnelles vécues sont encryptées dans des non-dits, le déni des pères repérable à chaque génération. C'est une inclusion, une incorporation, par la génération, d'une sorte d'enclave qui accentue la perméabilité transgénérationnelle. Faire le deuil de sa mère en thérapie est pour Sylvie l'occasion d'une première figuration, certes fétichique, d'un deuil antérieur non fait et sur lequel pèse un secret antilibid inal. Dans cette configuration, le père mort gît encrypté. Si le mort porte le masque du survivant, ce dernier renferme en lui le caveau d'un indicible transgénérationnel. La souffrance de Sylvie se révèle enfin au grand jour, " mon père était inexistant, il m'a manqué, il m'a manqué, il m'a manqué », exprime-t-elle de façon scandée pour souligner l'intensité de l'absence paternelle.

\section{CONCLUSION}

Le syndrome de Mü nchhausen présente des caractéristiques communes intergénérationnelles,

\footnotetext{
11 P.-C. Racamier, «Letravail des secrets : préliminaires », Groupal, 2, Paris, Les Éditions du collège de psychanalyse groupale et familiale, 1996, p. 109.

12 J. Guyotat, Mort, naissance et filiation : études de psychopathologie sur le lien de filiation, Paris, Masson, 1980.
} 
occasionne un déni de l'origine, des clivages, une angoisse de différenciation et de séparation. La dangerosité maternelle et la non-temporisation par un père n'ont pas permis l'instauration d'un sentiment d'exister par soi-même dans une présence dans l'absence, source du sentiment de continuité et base d'une assise narcissique. La fragilité des assises identificatoires de Mme M. par rapport à l'héritage maternel l'incite à chercher un fétiche, pouvant la soutenir narcissiquement. Ce transgénérationnel, inélaborable, de l'ordre de la crypte hantée par le père mort, fige toute la famille dans des liens fusionnels/confusionnels et dans une grande destructivité, au prix d'une opération " sacrificielle ", projetée sur l'enfant instrumentalisé. Le père incarné dans la personne du médecin pourrait ainsi interférer dans le couple mère-enfant pathologique afin qu'une séparation moi-objet puisse s'établir, que la suppression de l'emprise toute-puissante cesse et que les influences mortifères disparaissent, par la loi qui aurait fait défaut durant l'enfance maternelle. Les limites du langage ne pouvant exprimer l'indicible, la métaphore du vampirisme s'applique pour vaincre ces limites. Ce corps à corps questionne la féminité et les difficultés de passage au maternel, en relation avec la problématique préoedipienne. La pathologie de l'enfant devient l'aspiration essentielle et la condition d'un épanouissement.

Le syndrome de Münchhausen combine le $\quad s$ pulsions maternelles mortifères transgénérationnelles aboutissant à l'infanticide, au matricide et au parricide. L'emprise permet l'accès à un pouvoir total, la conquête de l'immortalité et la jouis sance extrême. Le temps s'arrête et signifie un retour au même, une boucle sur sa propre origine et une renaissance par enfant interposé mais aussi un retour au non-être et à la mort. La castration symbolique, le langage et les fantasmes oedipiens sont écartés. Le vampirisme s'inscrit dans le champ des recherches sur l'archaïque intergénérationnel et tente de figurer l'irreprésentable. Ces traumatis mes ont été éprouvés sans être reconnus et ces traces mnésiques désaffectées sont demeurées dépourvues de sens. Sylvie se trouve donc dans le rôle méd iateur de conflits intrapsychiques maternels par la délégation. Elle est envahie par la mère dont elle dépend et s'efface corps et âme dans la collusion du déni, dans la répression de son expression et dans ses capacités propres de représentation. Nous soutenons que le mythe du vampirisme dévoile un processus psychique qui est de l'ordre de l'emprise par le réel et pourrait devenir un nouveau concept opérationnel des pathologies d'expression corporelle comme dans « le syndrome de Mü nchhausen par procuration ».

\section{BIBLIOGRAPHIE}

Abraham, N. ; ToroK, M. 1987. L'écorce et le noyau, Paris, Flammarion.

Berger, M. 1997. «Pathologie du lien primaire et séduction narcissique », Groupal,

3, «L'incestuel », Paris, Les Éditions du collège de p sychanaly se groupale et

familiale.

DSM-IV-TR. 2002. Manuel diagnostique et sta tistique des troubles mentaux, Issy-les-Moulineaux,

Elsevier-Masson.

FÉDIDA, P. 2001. Des bienfaits de la dépression : éloge de la psychothérapie, Paris, Odile

Jacob.

GuYotat, J. 1980. Mort, naissance et filiation : études de psychopathologie sur le lien de

filiation, Paris, Masson.

KRISTEVA, J. 1987. Soleil noir : dépression et mélancolie, Paris, Gallimard.

Le Nestour, A. ; RAPhä̈l, A.M. ; RAPhä̈L, S. ; Golberine, G. 1999. «Infanticides

sous surveillance médicale », Sciences et avenir, $\mathrm{n}^{\circ} 2667$.

LyONS, R. ; M ASTERS, N. ; WU, J. 1991. « Issues in the identification and management

of Münchhausen syndrome by proxy », Infant Mental Health Journal, 12, 4,

Michigan, Michigan Association for Infant Mental Health.

RACAMIER, P.-C. 1996. « Le travail des secrets : préliminaires », Groupal, 2, Paris, Les

Éditions du collège de psychanaly se groupale et familiale.

Wolf-FÉdIDA, M. 2005. Amour, identité et changement, Paris, MJW Fédition.

Résumé

Le syndrome de Münchhausen par procuration plonge ses racines dans un passé obscur, encore opacifié par l'identification au père mort, qui caractérise cette pathologie dépressive. L'unité duelle avec une mère vampirique évoque l'idée d'une séparation-non-séparation, la compulsion de répétition et son au-delà, et le passage d'une génération à une autre. Les sentiments ambivalents pour le père sont réorientés vers un docteur qui agit sans le savoir en tant que parent symbolique et qui se retrouvent mis au défi. La mise en échec du savoir médical, l'image de la mère modèle sont autant de profits psychologiques qui concourent à combler la faille narcissique. Ces transactions impliquent également une distorsion des paramètres temporels puisque le temps générationnel est à la fois suspendu et accéléré, avec un risque de compulsion de répétition transgressive. 
Vampirisme, syndrome de Münchhausen par procuration, emprise, transgénérationnel, désubjectivation.

The DEAd SILENCE OFMüNCHHAUSEN Syndrome By ProXy

Summary

Münchhausen syndrome by proxy is rooted in a dark past , still clouded by identification with the father died, which characterizes the depressive illness. The dual unity evokes the idea of separation, non-separation, the repetition compulsion and beyond, and the transition from one generation to another. Ambivalent feelings for the father are redirected to a doctor who is unknowingly as a parental figure and find themselves challenged. The thwarting of medical knowledge, the image of the mother as much profit model are psychological which contribute to fill the narcissistic vulnerability. These transactions also involve a distortion of the temporal parameters as generational time is both suspended and accelerated, with a risk of répétition compulsion transgressive.

Keywords

Vampirism, Münchhausen syndrome by proxy, predominant influence, transgenerationnal,

desubjectivation. 